\title{
Interpenetration as a Mechanism for Negative Thermal Expansion in the Metal-Organic Framework $\mathrm{Cu}_{3}(\mathbf{b t b})_{2}$ (MOF-14)**
}

\author{
Yue Wu, Vanessa K. Peterson, Emily Luks, Tamim A. Darwish, and Cameron J. Kepert*
}

\begin{abstract}
Metal-organic framework materials (MOFs) have recently been shown in some cases to exhibit strong negative thermal expansion (NTE) behavior, while framework interpenetration has been found to reduce NTE in many materials. Using powder and single-crystal diffraction methods we investigate the thermal expansion behavior of interpenetrated $\mathrm{Cu}_{3}(\mathrm{btb})_{2}(\mathrm{MOF}-14)$ and find that it exhibits an anomalously large NTE effect. Temperature-dependent structural analysis shows that, contrary to other interpenetrated materials, in MOF-14 the large positive thermal expansion of weak interactions that hold the interpenetrating networks together results in a low-energy contractive distortion of the overall framework structure, demonstrating a new mechanism for NTE.
\end{abstract}

Negative thermal expansion (NTE) - contraction upon warming and expansion upon cooling-is a property that has been observed in a relatively small number of materials, with its occurrence over a broad temperature range being particularly rare. ${ }^{[1]}$ A number of different mechanisms have been demonstrated to favor this unusual property, which principally include various electronic and magnetic transitions, ${ }^{[2]}$ and transverse atomic and molecular vibrations. ${ }^{[3-9]}$ NTE materials that exploit the latter mechanism include metal oxides such as $\mathrm{ZrW}_{2} \mathrm{O}_{8},{ }^{[4,10]}$ various zeolites, ${ }^{[5]}$ and a growing family of metal cyanides. ${ }^{[6-8,11]}$ In these openframework systems, increased transverse "skipping rope type" motion of the oxide and cyanide linkers between metal centers upon warming leads to bulk contraction. Framework connectivity strongly affects the magnitude of

[*] Y. Wu, Prof. C. J. Kepert

School of Chemistry, The University of Sydney

Sydney NSW 2006 (Australia)

E-mail: cameron.kepert@sydney.edu.au

Homepage: sydney.edu.au/science/chemistry/ cjkgroup/

Dr. V. K. Peterson, E. Luks, Dr. T. A. Darwish

Bragg Institute, Australian Nuclear Science \& Technology Organisation (Australia)

$[* * *$ This work was supported by research and fellowship funding from the Australian Research Council (C.J.K.), and Post-Graduate Research Awards (Y.W.) from the Australian Government and the Australian Institute of Nuclear Science and Engineering (AINSE). Work was undertaken at the Australian Synchrotron MX-1 (M2200) and PD beamlines (M2919) and at the Advanced Photon Source 1. BM-C beamline (GUP-12462). Use of the Advanced Photon Source, an Office of Science User Facility operated for the U.S. Department of Energy (DOE) Office of Science by Argonne National Laboratory, was supported by the U.S. DOE under Contract No. DE-AC02$06 \mathrm{CH} 11357$. We thank Gregory J. Halder for beamline support. Supporting information for this article is available on the WWWW under http://dx.doi.org/10.1002/anie.201311055.
NTE in these systems, with the more weakly bonded, doubleatom-bridged cyanide frameworks generally showing greater NTE than the more strongly bonded, single-atom-bridged oxide frameworks, as seen for example in the observation of pronounced isotropic NTE in single-network $\mathrm{Cd}(\mathrm{CN})_{2}{ }^{[6]}$ Low energy transverse vibrations have also been shown to lead to highly anisotropic behaviors within these materials families, ${ }^{[12]}$ an extreme example being the colossal thermal expansion and contraction seen in $\mathrm{Ag}_{3} \mathrm{Co}(\mathrm{CN})_{6}$ corresponding to thermally induced hingeing of the framework associated with atom-atom repulsion. ${ }^{[12]}$

Most recently, NTE has been found to occur in certain metal-organic frameworks (MOFs) due to their combination of flexible metal coordination polyhedra, elongated molecular linkers and open pore spaces. ${ }^{[13-17]}$ The greater structural complexity of these materials compared to the oxide and cyanide framework systems is paralleled by their considerably more complex structural dynamics, allowing the potential for new vibrational mechanisms for NTE to be discovered and developed. In $\mathrm{Cu}_{3}(\mathrm{btc})_{2}$ (btc $=1,3,5$-benzenetricarboxylate), for example, NTE arises uniquely from the transverse motion of planar rather than linear linkers and, in addition, from the local dynamic deformation of $\mathrm{Cu}_{2}$ (carboxylate) "paddlewheel" nodes within its framework structure. ${ }^{[13,14]}$ Similar complexity of behavior, involving both local and long-range modes, has also been found in MOF-5 and related phases. ${ }^{[15,16]}$

With an interest in further extending the search for novel NTE framework dynamics in MOFs, our attention has been drawn to the interpenetrated framework $\mathrm{Cu}_{3}(\mathrm{btb})_{2}$ (MOF-14; $\mathrm{btb}=4,4^{\prime}, 4^{\prime \prime}$-benzene-1,3,5-triyl-tribenzoate $)^{[18]}$ due to it sharing some structural similarities with $\mathrm{Cu}_{3}(\mathrm{btc})_{2}$ and to the intriguing observation that as an interpenetrated phase its volume is reduced from that of the corresponding singlenetwork phase of the same formula, MOF-143. ${ }^{[19]}$ Here we show that this material exhibits pronounced NTE over a broad temperature range-an observation that contrasts with previous findings that framework occlusion associated with interpenetration and the presence of guest species hinders or even completely masks $\mathrm{NTE}^{[6,9]}$ due to the influence of physical obstructions on the energy and amplitude of transverse NTE vibrations. Moreover, through analysis of the temperature-dependent structure we delineate a new mechanism for NTE in which repulsion between the two interpenetrating networks causes their progressive geometric distortion upon warming.

To investigate the thermal expansion behavior of MOF14 , evacuated samples were characterized by a combination of X-ray and neutron powder and X-ray single crystal diffraction techniques, leading to the determination of con- 


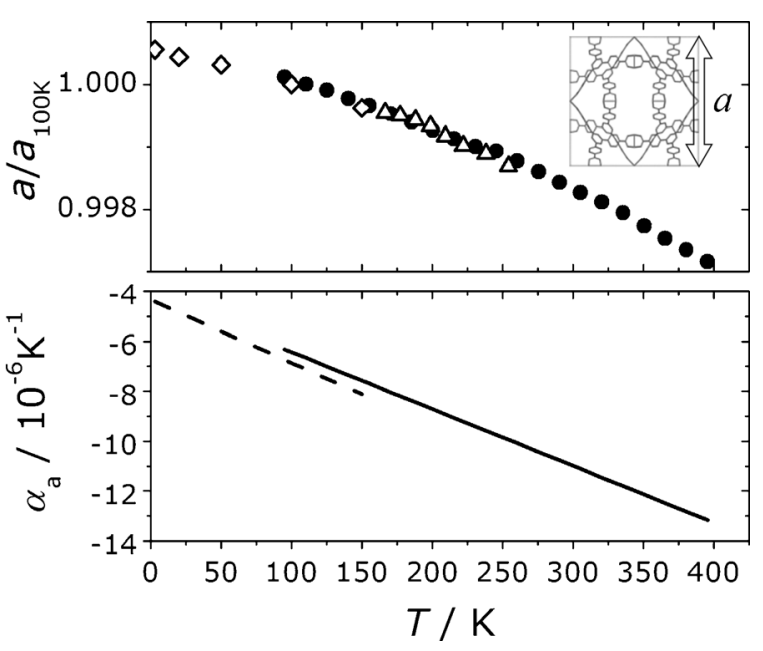

Figure 1. Top: Temperature-dependent fractional unit-cell evolution of MOF-14 obtained by powder neutron diffraction (hollow diamonds), synchrotron powder X-ray diffraction (black circles) and synchrotron single-crystal X-ray diffraction (hollow triangles). Due to absolute value offsets, all values are normalized to $100 \mathrm{~K}$. Inset: Structural picture of the cubic interpenetrated structure. Bottom: Temperature-dependent coefficients of thermal expansion determined from fits to the neutron (dashed) and X-ray (solid) powder data.

tinuous, monotonic NTE over a broad temperature range (Figure 1). The temperature-dependent coefficient of thermal expansion $\left(\alpha_{1}=\mathrm{d} a / a \mathrm{~d} T\right)$ was determined using second-order polynomial fits to each set of powder diffraction data, from which it was found that $\alpha_{1}$ increases in magnitude from -4 to $-13 \times 10^{-6} \mathrm{~K}^{-1}$ over the temperature range 3 to $400 \mathrm{~K}$. This magnitude of NTE notably extends to more than three times that of $\mathrm{Cu}_{3}(\mathrm{btc})_{2}$, for which $\alpha_{1}=-4.1(1) \times 10^{-6} \mathrm{~K}^{-1}$ over the range 100 to $500 \mathrm{~K}^{[13]}$ In general terms this increase is unexpected given that previous work shows that, while framework materials with more flexibility are expected to show more pronounced NTE ${ }^{[8]}$ framework interpenetration tends to dramatically diminish NTE due to the steric obstruction of transverse vibrational modes. ${ }^{[6]}$

In considering the reason for the pronounced NTE observed, it is instructive to firstly compare the structure of MOF-14 with that of its non-interpenetrated analogue, MOF143. The cubic unit cell parameter of solvated MOF-143 $3^{[18]}$ (note that the crystallinity of this phase is destroyed upon guest desorption) is ca. $2 \%$ larger than any of the solvated or desolvated forms of MOF-14 reported or presented herein. As chemical connectivity of the individual MOF-14 networks is identical to that of MOF-143, the smaller cell of MOF-14 arises from a structural distortion resulting from its framework interpenetration. Specifically, whereas MOF-143 closely fits the ideal pto (platinum-oxide type) net for a framework consisting of trigonal and square planar linking units, ${ }^{[20]}$ in MOF-14 the btb moieties distort outwards on either side of their ideal positions to accommodate each other. This reduces the distance between the coordinating arms of the btb, drawing adjacent paddlewheels closer together and causing the difference in observed unit cell parameters between the two materials. In light of this observation, there is some expectation that the steric mechanism responsible for this ca.
$6 \%$ volume contraction upon interpenetration may increase in magnitude with increasing temperature.

To elucidate the structural dynamics responsible for the unusually large NTE effect observed, a detailed analysis of the temperature-dependent structure was undertaken. The two interpenetrating networks in MOF-14 are held in place by multiple attractive edge-to-face $\mathrm{C}-\mathrm{H} \cdots \pi$ interactions between neighboring btb moieties. At all temperatures, the centroids of the peripheral phenyl rings lie at distances of ca. $3.0 \AA$ and $4.1 \AA$ from the nearest $\mathrm{H}$ atoms. In contrast, the central phenyl rings have a face-to-face arrangement that has been shown to give a repulsive $\pi \cdots \pi$ interaction. ${ }^{[21]}$ Single crystal structural analyses show that the distance between the two central phenyl rings increases markedly with temperature (Figure 2). Concurrently, the tilt of the peripheral phenyl
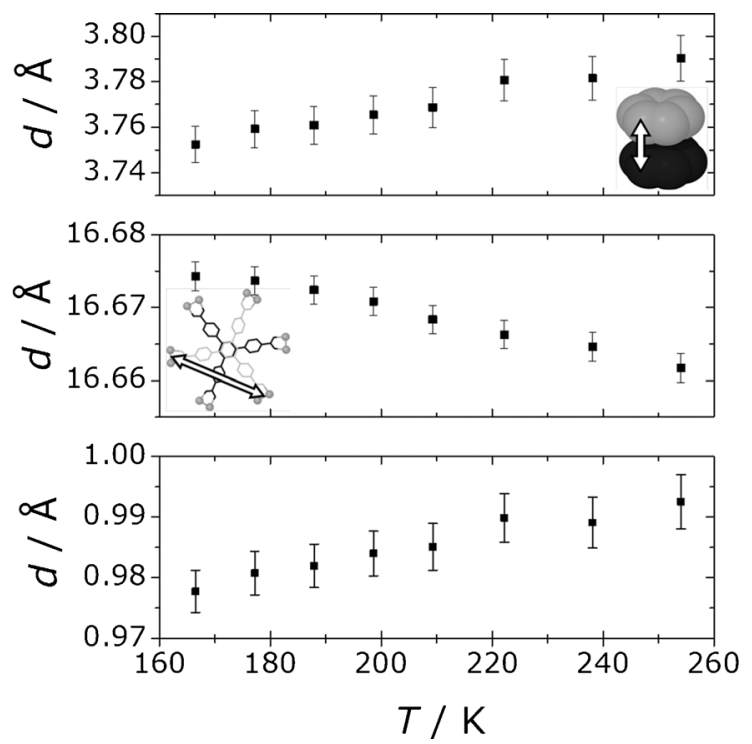

Figure 2. Top: Thermal expansion of the distance between the central phenyl fragments of the btb ligands. Middle: Thermal contraction of the distance between adjacent paddlewheel centroids. Center inset: $A$ fragment of the MOF-14 crystal structure, showing the relative positions of the btb moieties from two networks. Bottom: Temperature-dependent change of the distance between the plane of the btb central phenyl ring and the plane of the three connected paddlewheel centroids.

rings increases (Figure S4) such that the interaction between the neighboring rings is closer to the ideal $\mathrm{C}-\mathrm{H} \cdots \pi$ configuration. The overall effect is to buckle the two moieties outwards from each other, drawing the connected paddlewheel units closer in (Figure 2; effect shown diagrammatically in Figure 3). Due to the highly porous nature of the material, there is little steric obstruction of these distortions.

The crystallographic results also demonstrate the distortive NTE mechanism at the unit-cell scale. The MOF-14 structure has one main large pore in the center of each cubic unit cell. This pore is defined by a cage of eight btb moieties forming the "panels" of the cage, joined at ten approximately right angle paddlewheel vertices (Figure 4 left). The btb faces of this cage are concave (they bulge inward into the pore). 

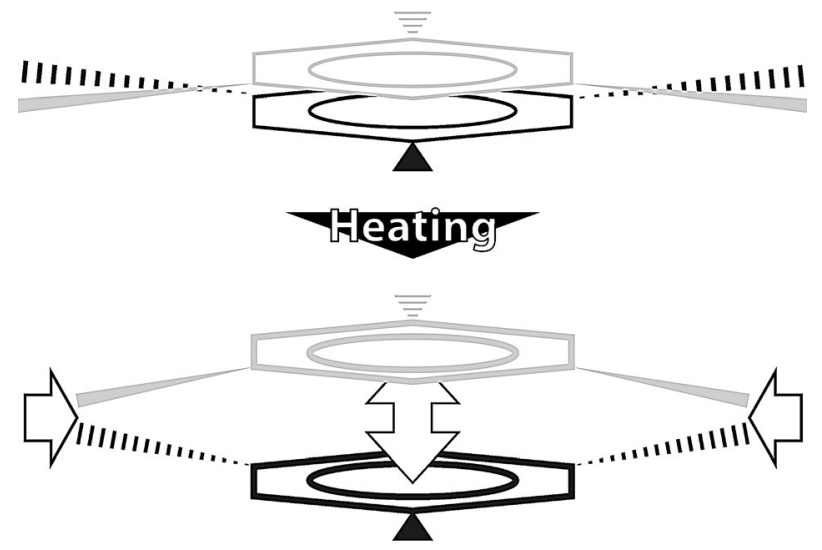

Figure 3. Diagram showing an exaggeration of the contraction-inducing biconvex distortion of the face-to-face btb ligands.

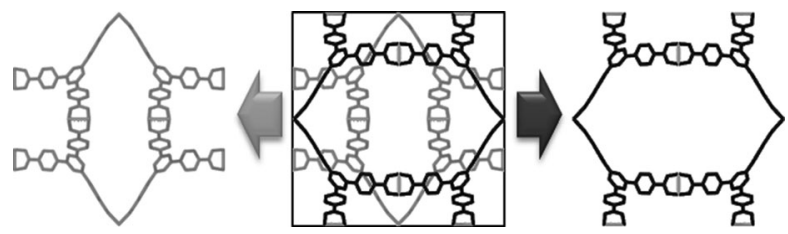

Figure 4. The arrangement of the interpenetrating nets in each MOF. 14 unit cell.

The other network present forms another cage of identical connectivity but orthogonal orientation around that pore; these btb moieties are convex to the pore, but also form the concave faces of the cages surrounding the adjacent pores (Figure 4 right). The change in the distance between carboxylate carbon atoms on the different cages (inset, Figure 5) in each network shows how the overall framework contraction results from the structurally induced distortion. The "concave" cage shows a contraction (open circle data, Figure 5), while the "convex" cage shows no significant expansion (filled squares). As these two distances sum to give the unit cell parameter, the net result is an overall framework contraction. Locally, we can consider each btb unit as undergoing low

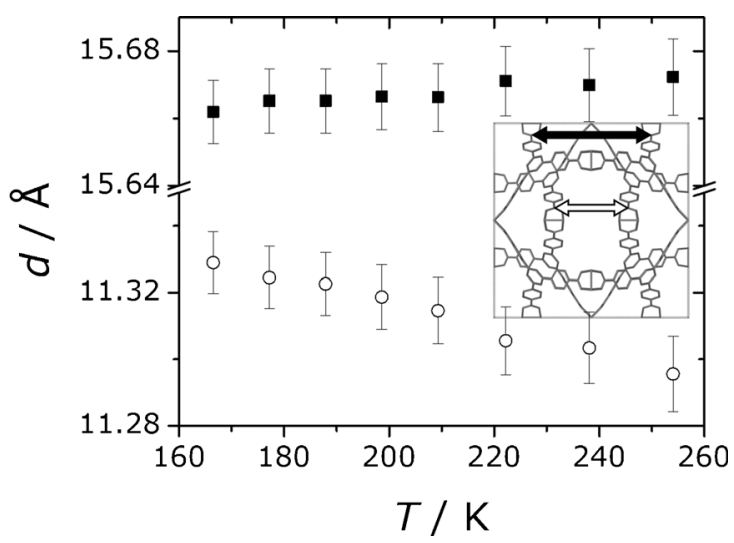

Figure 5. Temperature-dependent distance between the intra-framework carboxylate carbon atoms. energy transverse motion in a highly asymmetric potential well, bound by neighboring btb on one side and vacant pore space on the other. This situation is broadly akin to the $\mathrm{Ag} \cdots \mathrm{Ag}$ repulsion present in $\mathrm{Ag}_{3} \mathrm{Co}(\mathrm{CN})_{6},{ }^{[12]}$ with resulting colossal anisotropic NTE and PTE along perpendicular axes, although here occurs within a cubic framework and therefore yields isotropic NTE behavior.

To conclude, we delineate a new vibrational mechanism for NTE in which increased framework distortion associated with interframework repulsion leads to pronounced isotropic contraction upon warming. This "distortive" mechanism for NTE stands in contrast to that present in many other NTE materials, in which transverse vibrations occur within highly symmetric environments and so do not yield a strongly distortive component. This result suggests a new method by which positive thermal expansion may be counteracted, exploiting the thermal expansion of weak interactions to drive contractive low-energy framework distortions.

\section{Experimental Section}

$\mathrm{Cu}_{3}(\mathrm{btb})_{2}$ was synthesized according to the procedure of Chen et al. ${ }^{[18]} \mathrm{H}_{3} \mathrm{btb}(0.023 \mathrm{~g}, 0.052 \mathrm{mmol})$ and $\mathrm{Cu}\left(\mathrm{NO}_{3}\right)_{2} \cdot 2.5 \mathrm{H}_{2} \mathrm{O}(0.065 \mathrm{~g}$, $0.28 \mathrm{mmol}$ ) were allowed to react in a solvent mixture of ethanol $(3 \mathrm{~mL}), N, N^{\prime}$-dimethylformamide (DMF) $(3 \mathrm{~mL})$, and water $(2 \mathrm{~mL})$ in the presence of excess pyridine $(0.62 \mathrm{mmol})$ at $65^{\circ} \mathrm{C}$ for 1 day, giving light blue cubic crystals. All samples for analysis were washed in ethanol then desolvated overnight under vacuum at room temperature. Deuterated samples for neutron diffraction were prepared following the above synthesis, with all reagents scaled up 20 times. The fully deuterated btb ligand was prepared at the ANSTO National Deuteration Facility.

Single-crystal X-ray diffraction data were collected at the Australian Synchrotron MX-1 beamline. The beamline provided silicon double crystal monochromated radiation $(\lambda=0.77487 \AA)$ and was equipped with an ADSC Quantum 210r detector and Cryojet cooling system. Structural collections were taken using a $360^{\circ} \mathrm{Phi}$ scan. The Blu-Ice interface was used for data collection. ${ }^{[2]}$ The XDS package was used for data reduction. ${ }^{[23]}$ For structure solution and refinement the SHELX-97 package was used through the X-Seed interface ${ }^{[24]}$ CCDC $960248-960258$ contain the supplementary crystallographic data for this paper. These data can be obtained free of charge from The Cambridge Crystallographic Data Centre via www. ccdc.cam.ac.uk/data_request/cif.

Variable-temperature synchrotron PXRD data were collected at the Advanced Photon Source 1-BM-C beamline $(\lambda=0.60941 \AA)$ using a mar345 image plate detector, where collections were taken continually while heating at $120 \mathrm{~K} \mathrm{~h}^{-1}$ from $100 \mathrm{~K}$ until the sample decomposed. Data were also collected at the Australian Synchrotron PD beamline $(\lambda=0.79941 \AA)$, using a Mythen microstrip detector. Stepwise heating was applied, with the temperature held constant for each collection. ${ }^{[25]}$ PND data were collected on a $0.48 \mathrm{~g}$ sample for $12 \mathrm{~h}$ on the ECHIDNA beamline at the OPAL Reactor $(\lambda=$ $2.4408 \AA$ ).

Received: December 20, 2013

Published online: April 1, 2014

Keywords: mechanical properties - metal-organic frameworks . negative thermal expansion - structural dynamics . structure elucidation 
[1] A. W. Sleight, Curr. Opin. Solid State Mater. Sci. 1998, 3, 128 131; J. S. O. Evans, J. Chem. Soc. Dalton Trans. 1999, 3317-3326; C. Lind, Materials 2012, 5, 1125-1154.

[2] Y. Hao, Y. Gao, B. Wang, J. Qu, Y. Li, J. Hu, J. Deng, Appl. Phys. Lett. 2001, 78, 3277-3279; J. R. Salvador, F. Guo, T. Hogan, M. G. Kanatzidis, Nature 2003, 425, 702-705; J. Arvanitidis, K. Papagelis, S. Margadonna, K. Prassides, A. N. Fitch, Nature 2003, 425, 599-602; K. Takenaka, H. Takagi, Appl. Phys. Lett. 2005 87, 261902-261903.

[3] V. Korthuis, N. Khosrovani, A. W. Sleight, N. Roberts, R Dupree, W. W. Warren, Jr., Chem. Mater. 1995, 7, 412-417; J. S. O. Evans, Z. Hu, J. D. Jorgensen, D. N. Argyriou, S. Short, A. W. Sleight, Science 1997, 275, 61-65; C. Lind, A. P. Wilkinson, Z. Hu, S. Short, J. D. Jorgensen, Chem. Mater. 1998, 10 , 2335-2337; J. S. O. Evans, J. C. Hanson, A. W. Sleight, Acta Crystallogr. Sect. B 1998, 54, 705-713; K. W. Chapman, P. J. Chupas, C. J. Kepert, J. Am. Chem. Soc. 2005, 127, 15630-15636.

[4] T. A. Mary, J. S. O. Evans, T. Vogt, A. W. Sleight, Science 1996 $272,90-92$.

[5] P. Lightfoot, D. A. Woodcock, M. J. Maple, L. A. Villaescusa, P. A. Wright, J. Mater. Chem. 2001, 11, 212-216.

[6] A. E. Phillips, A. L. Goodwin, G. J. Halder, P. D. Southon, C. J. Kepert, Angew. Chem. 2008, 120,1418-1421; Angew. Chem. Int. Ed. 2008, 47, 1396-1399.

[7] K. W. Chapman, P. J. Chupas, C. J. Kepert, J. Am. Chem. Soc. 2006, 128, 7009-7014.

[8] A. L. Goodwin, C. J. Kepert, Phys. Rev. B 2005, 71, 140301.

[9] A. L. Goodwin, K. W. Chapman, C. J. Kepert, J. Am. Chem. Soc. 2005, 127, 17980-17981.

[10] G. Ernst, C. Broholm, G. R. Kowach, A. P. Ramirez, Nature 1998, 396, 147-149.

[11] J. L. Korčok, M. J. Katz, D. B. Leznoff, J. Am. Chem. Soc. 2009, 131，4866-4871; S. G. Duyker, V. K. Peterson, G. J. Kearley, A. J. Ramirez-Cuesta, C. J. Kepert, Angew. Chem. 2013, 125 , 5374-5378; Angew. Chem. Int. Ed. 2013, 52, 5266-5270; T. Pretsch, K. W. Chapman, G. J. Halder, C. J. Kepert, Chem. Commun. 2006, 1857-1859; S. J. Hibble, A. M. Chippindale, E. Marelli, S. Kroeker, V. K. Michaelis, B. J. Greer, P. M. Aguiar, E. J. Bilbé, E. R. Barney, A. C. Hannon, J. Am. Chem. Soc. 2013, 135, 16478-16489; R. Mittal, M. Zbiri, H. Schober, E. Marelli,
S. J. Hibble, A. M. Chippindale, S. L. Chaplot, Phys. Rev. B 2011, 83, 024301.

[12] A. L. Goodwin, M. Calleja, M. J. Conterio, M. T. Dove, J. S. O. Evans, D. A. Keen, L. Peters, M. G. Tucker, Science 2008, 319, $794-797$.

[13] Y. Wu, A. Kobayashi, G. J. Halder, V. K. Peterson, K. W. Chapman, N. Lock, P. D. Southon, C. J. Kepert, Angew. Chem. 2008, 120, 9061-9064; Angew. Chem. Int. Ed. 2008, 47, 89298932.

[14] V. K. Peterson, G. J. Kearley, Y. Wu, A. J. Ramirez-Cuesta, E. Kemner, C. J. Kepert, Angew. Chem. 2010, 122, 595-598; Angew. Chem. Int. Ed. 2010, 49, 585-588.

[15] N. Lock, M. Christensen, Y. Wu, V. K. Peterson, M. K. Thomsen, R. O. Piltz, A. J. Ramirez-Cuesta, G. J. McIntyre, K. Noren, R. Kutteh, C. J. Kepert, G. J. Kearley, B. B. Iversen, Dalton Trans. 2013, 42, 1996-2007.

[16] N. Lock, Y. Wu, M. Christensen, L. J. Cameron, V. K. Peterson, A. J. Bridgeman, C. J. Kepert, B. B. Iversen, J. Phys. Chem. C 2010, 114, $16181-16186$.

[17] D. Dubbeldam, K. S. Walton, D. E. Ellis, R. Q. Snurr, Angew. Chem. 2007, 119, 4580-4583; Angew. Chem. Int. Ed. 2007, 46, 4496-4499; S. S. Han, W. A. Goddard, J. Phys. Chem. C 2007, 111, 15185-15191; W. Zhou, H. Wu, T. Yildirim, J. R. Simpson, A. R. Hight Walker, Phys. Rev. B 2008, 78, 054114.

[18] B. Chen, M. Eddaoudi, S. T. Hyde, M. O'Keeffe, O. M. Yaghi, Science 2001, 291, 1021-1023.

[19] H. Furukawa, Y. B. Go, N. Ko, Y. K. Park, F. J. Uribe-Romo, J. Kim, M. O'Keeffe, O. M. Yaghi, Inorg. Chem. 2011, 50, $9147-$ 9152.

[20] S. Amirjalayer, M. Tafipolsky, R. Schmid, J. Phys. Chem. C 2011, $115,15133-15139$.

[21] C. Janiak, J. Chem. Soc. Dalton Trans. 2000, 3885-3896.

[22] T. M. McPhillips, S. E. McPhillips, H.-J. Chiu, A. E. Cohen, A. M. Deacon, P. J. Ellis, E. Garman, A. Gonzalez, N. K. Sauter, R. P. Phizackerley, S. M. Soltis, P. Kuhn, J. Synchrotron Radiat. 2002, $9,401-406$.

[23] W. Kabsch, Acta Crystallogr. Sect. D 2010, 66, 125-132.

[24] G. M. Sheldrick, Acta Crystallogr. Sect. A 2008, 64, 112-122; L. J. Barbour, J. Supramol. Chem. 2001, 1, 189-191.

[25] K. S. Wallwork, B. J. Kennedy, D. Wang, AIP Conf. Proc. 2007, $879,879-882$ 